This item was submitted to Loughborough's Research Repository by the author.

Items in Figshare are protected by copyright, with all rights reserved, unless otherwise indicated.

\title{
Review of selection criteria for sensor and actuator configurations suitable for internal combustion engines
}

PLEASE CITE THE PUBLISHED VERSION

https://doi.org/10.4271/2018-01-0758

\section{PUBLISHER}

(C) SAE International

VERSION

AM (Accepted Manuscript)

\section{PUBLISHER STATEMENT}

This work is made available according to the conditions of the Creative Commons Attribution-NonCommercialNoDerivatives 4.0 International (CC BY-NC-ND 4.0) licence. Full details of this licence are available at: https://creativecommons.org/licenses/by-nc-nd/4.0/

\section{LICENCE}

CC BY-NC-ND 4.0

\section{REPOSITORY RECORD}

Comissiong, Rhys M., and Thomas Steffen. 2018. "Review of Selection Criteria for Sensor and Actuator Configurations Suitable for Internal Combustion Engines". figshare. https://hdl.handle.net/2134/34310. 


\title{
Review of Selection Criteria for Sensor and Actuator Configurations Suitable for Internal Combustion Engines
}

\author{
Rhys Comissiong and Thomas Steffen \\ Loughborough University
}

\begin{abstract}
This literature review considers the problem of finding a suitable configuration of sensors and actuators for the control of an internal combustion engine. It takes a look at the methods, algorithms, processes, metrics, applications, research groups and patents relevant for this topic. Several formal metric have been proposed, but practical use remains limited. Maximal information criteria are theoretically optimal for selecting sensors, but hard to apply to a system as complex and nonlinear as an engine. Thus, we reviewed methods applied to neighboring fields including nonlinear systems and nonminimal phase systems. Furthermore, the closed loop nature of control means that information is not the only consideration, and speed, stability and robustness have to be considered. The optimal use of sensor information also requires the use of models, observers, state estimators or virtual sensors, and practical acceptance of these remains limited. Simple control metrics such as conditioning number are popular, mostly because they need fewer assumptions than closed-loop metrics, which require a full plant, disturbance and goal model. Overall, no clear consensus can be found on the choice of metrics to define optimal control configurations, with physical measures, linear algebra metrics and modern control metrics all being used. Genetic algorithms and multi-criterial optimisation were identified as the most widely used methods for optimal sensor selection, although addressing the dimensionality and complexity of formulating the problem remains a challenge. This review does present a number of different successful approaches for specific applications domains, some of which may be applicable to diesel engines and other automotive applications. For a thorough treatment, non-linear dynamics and uncertainties need to be considered together, which requires sophisticated (non-Gaussian) stochastic models to establish the value of a control architecture.
\end{abstract}

\section{Introduction}

Current design processes of internal combustion engines are based on experience and incremental improvements, which may not lead to the optimal control architecture. Engines are designed to meet certain emissions regulations and performance criteria, subject to cost constraints. As with any trade-off, certain sensors and actuators can cause the engine to perform optimally for one subsystem, like aftertreatment, but can inhibit the performance for another, such as air system control. Various sensors measure variables such as speed, pressure and temperature to maintain torque and emissions control. However, often the systems controlling them are legacy-based implementation rather than utilising optimised control methods. We aim to find optimised control systems based on assessment metrics including degrees of freedom, open-loop performance, closed-loop performance and other metrics related to controllability/observability. The issue of optimal control architecture is not exclusive to engines and is applicable to many different areas of engineering. We aim to review the decisions and methods used to select sensors and actuators for different applications. The wider goal of this work is to identify potential areas of research that can be used to design suitable sensor and actuator configurations for different design trade-offs. This can potentially lead to the reduction of physical sensors and actuators used on engines and other applications.

\section{Review of Sensor and Actuator Configurations Selection Criteria Methods}

\section{Methodology}

Initially, search terms were divided into 3 sections. Firstly, Sensors and Actuators, which included Sensor Signal Processing and Actuator, combined with the terms Virtual, Placement, Selection, Fit, Set, Observability, and Detectability. Secondly the list of Control Metric search terms included: Fault Tolerant Sensor, Object Oriented Architecture Control, Sensor Redundancy, Plant Domain Control, Input-Output Methods Control, Controllability, Right Half Plane Zeros, Manipulation and Estimation, Robust Stability, Maximum Entropy Sensors, and Optimal Sensor Placement Industry. Finally, the terms Actuator, Signal Processing and Sensor, were combined with the industrial search terms: Internal Combustion Engine, Power Plants, Process Control, Automotive and Aeronautical.

For the searches, the following databases were used due to the flexible search capabilities (we focused mainly on the first two due to their relevance within the field):

- Institute of Electronic and Electrical Engineers (IEEE)

- $\quad$ Society of Automotive Engineers (SAE)

- National Aeronautics and Space Administration (NASA)

- Web of Science

- Scopus

- Compendex

The initial search terms had demonstrated where literature could be found, however it did not give any indication of what could be learnt from the literature, or the level of relevance of the results. To deal with this problem an alternative form of filtering was applied. A question based filter was created to ensure that the information contained in the review answers these questions. The questions of the filter were:

1. Which industries use sensor/actuator selection filters?

2. Which methods are used in sensor/actuator selection?

3. What are the specific challenges of engine control?

4. How much data can you get through sensor/actuator signal processing?

5. Optimisation - What does it mean to be better?

6. Which specific sensors/actuators are suitable?

7. How do you define a good sensor/actuator?

Page 1 of 16 
8. Specific examples of where sensors/actuators are being used in industry?

After using the question based filter, the literature was then organized into four main areas: Industrial Uses, System Models, Optimization and Control Metrics. Initially we focused on recent literature to assess current selection methods used, and followed this up by reviewing older literature to complete the assessment of the questions.

Doctorate documents were not included as part of this review. Additionally, this review does not include an assessment of the economic costs saved by doing this work. Typically, this is not discussed in the literature explicitly, as this information is usually confidential. Our aim is to give insight into what research could be done, but the full implications requires in-depth technical assessments to be carried out, so that the trade-offs between performance and costs, including effort, are understood.

\section{Industrial Applications}

This section outlines examples of industrial examples of where sensors and actuators have been selected for different applications. The selection criteria and development of the control architecture all differ, thus optimal selection methods could be applicable in these examples.

Onen et al [1] summarizes the design of a lower extremity exoskeleton (wearable robots), named walking supporting exoskeleton (WSE). There are three stages in the WSE design: mechanical design, actuator selection, and controller design. Actuators used in exoskeleton applications are required to provide high torques while operating in high speeds. Therefore, DC servomotors are used as actuators because of their compactness, lightweight design, energy efficiency and controllability. Many criteria are used to select actuators including torque-mass ratio, velocity, range of motion, and controllability.

Control of wireless actuators in the process industry faces many challenges including feedback latency and battery longevity. Blevins et al [2] demonstrate how traditional control methodologies can be modified to effectively work with general wireless communication and achieve control of a wireless valve. They propose a modified Proportional-Intergral-Derivative (PID) Controller known as PIDPlus. Blevins et al propose a unique time-to-apply field when sending the output value to a wireless valve. This field specifies a time in the future when the output value takes effect. The implied valve position is calculated using the target position communicated to the valve and the time specified when the valve should take action. The proposed concepts were successful in controlling an industrial size flow process with wireless sensors and actuators.

S Puliyakote et al [3] used signal processing tools on Acoustic Emission (AE) from engine cylinder liner to identify faults found in critical engine components. Faults from a small petrol engine were recreated such as the engine running with worn out piston rings and under lubricant oil level. The benefits of AE are that it is nonintrusive and has good signal to noise ratio. Additionally, there are many sources of $\mathrm{AE}$ within an engine including valve impacts, fluid flow, combustion and sliding and rolling contact. In their case an $\mathrm{AE}$ sensor was attached to the periphery of the cylinder liner to collect the signals. The signal was then successfully processed to emphasise the characteristics of the faults in lubricant oil studies.

$\mathrm{J}$ Xiaojing et al [4] proposed the general design plan of networked testing platform for internal combustion engine based on virtual instrumentation. The state parameters, transducers models, configuration plans and signal condition circuits were implemented. Two types of state parameters were used: structure and diagnosis. The structural parameters determine the technical condition, whereas Page 2 of 16 diagnosis parameters are the indexes which are contacted with the structure parameters and show the technical state of the structure. A simple PID controller is used to control the virtual instrumentation. Three parts were used in the design of the signal acquisition and condition system's hardware: Central Processing Unit, signal measurement and data conversion. At time of publication the design platform was considered 'prospective and open'.

S Mohanty et al [5] characterize a wireless MEMS capacitive accelerometer for Industrial ball mill applications. The selection parameters include demands that the sensor is capable of mounting on a rotating platform, with higher value of acceleration, sensitivity, and wide bandwidth of operation. Higher sensitivity causes problems for the range of acceleration and the bandwidth of operation. An assessment of the sensor principles is described as well as performance at different tilt angles and rotational speeds. M Raoufat et al [6] proposed a new approach to design fault-tolerant wide-area damping controllers (WADCs). Power system security is provided using actuator redundancy. When an actuator fails, mechanically or through miscommunication, the supervisory controller redistributes the control signals to the remaining actuators of the system. Multi-objective $\mathrm{H}-2$ and $\mathrm{H}-\infty$ optimization with linear matrix inequality pole placement region was used in the design of the WADC to achieve high damping performance. As part of the fault tolerance, virtual actuators (VAs) are designed to manage actuator failures without the need to redesign the nominal WADC. These are made using a reconfiguration block, which is controller independent and only needed to know that the actuator is unavailable.

$\mathrm{H}$ Husted et al [7] developed a new sensor that directly detects the particles passing through the diesel particulate filter (DPF) and estimates the cumulative particle flow. New threshold levels for diagnosis have required new approaches as opposed to using feedback from differential pressure sensing across the filter. Due to high temperatures in the after-treatment, requirements of the particulate matter (PM) sensor include: Wide operating temperature range, Rugged and robust to exhaust exposure, Quick time to sensor ready, Accurate, Features to support diagnostic requirements, Provision for regeneration (heater), Wide exhaust velocity range, Senses near centre of exhaust pipe, and Robust to water condensate exposure. Physical placement of the sensor is also critical for quick sensor operation as the farther back from the DPF it is, the longer the delay to reach the dew point temperature, which is due to the large thermal mass of the DPF.

J Alfaya et al [8] considered the problems of cooling and energy management by designing a multivariable robust controller for a onestage refrigeration cycle and performing controllability analysis. The objective of the system was to maintain the temperature of the cold room at a desired value. Therefore, its controlled variables include the superheating degree of refrigerant at the evaporator outlet and the outlet temperature of the evaporator secondary flux. The manipulated variables are the electronic expansion valve opening and the compressor speed. Multivariable linear models are identified at several operating points, and a nominal one is chosen to minimize uncertainty. Challenges in the design included the speed of response, consequently, the compressor, the expansion valve and the thermal behaviour of secondary fluxes are statically modelled, whereas, for heat exchangers dynamical models are developed. Alfaya designed an $\mathrm{H}-\infty$ controller, based on unstructured uncertainty, using the Mixed Sensitivity Problem approach.

Table 1. Summary of industrial uses and their applications. 


\begin{tabular}{|l|l|l|}
\hline Authors & Industry & Applications \\
\hline Onen [1] & Medical & Exoskeletons \\
\hline Blevins [2] & Process & Wireless Actuators \\
\hline Puliyatoke [3] & Automotive & Acoustic Emission \\
\cline { 1 - 1 } Xiaojing [4] & Automotive & Testing platform - Virtual Instrumentation \\
\cline { 1 - 1 } Mohanty [5] & Materials & MEMS Accelerometer \\
\cline { 1 - 1 } Raoufat [6] & Power Systems & Virtual Actuators \\
\hline Husted [7] & Automotive & Particulate Matter Sensor \\
\hline Alfaya [8] & Energy Management & Refrigeration System \\
\hline
\end{tabular}

Even though the applications described come from different industries, each example describes methods that could be applied to sensor selection in for internal combustion engines to at least some degree. Later we will assess optimisation techniques from the aeronautical industry, where optimal placement methods are described extensively.

\section{System Models}

In order to consider transducers, it is essential to understand how they interact with the process under control, and this is usually achieved with a system model. In this paper, three fundamental types of dynamic models are considered: State Space, Modal Domain and Physical Models.

\section{State Space}

Many of the papers reviewed investigated control in linear systems. This is probably due to the fact that simple linear models and simulations can be produced to verify the control strategies. Franzè et al [9] and Munz [10] et al apply their fault tolerance and sensor placement algorithms, respectively, to linear discrete-time systems. Lee [11] defines degrees of freedom of control in linear systems. The main issue with linear control is that it does not represent real systems well because too many assumptions are made, and generally automotive systems are nonlinear. A whole engine has too many degrees of freedom for different domains to be modelled linearly, however, individual sections at particular operating points can be modelled, and scheduled state space control techniques can be applied.

\section{Modal Domain}

Another system model that control analysis has been performed on in this area is in the modal domain. Frequency-based control has been extensively researched. In addition to linear systems, Lee [12] defines controllability metrics in the modal domain. These are in the form of controllability and observability Gramian matrices.

Automotive engine efficiency is significantly dependent on the performance of the sensors and actuators installed. Sliding Mode Observers (SMO) are widely used for fault diagnosis of dynamical systems. The key attribute of nonlinear SMO is its robust nature. However, multiple filters are used for state/parameter estimation, which may lead to corruption in results. Q Ahmed et al [13] proposed a Second Order Sliding Mode Observer for virtual sensors to monitor Air Intake System (AIS) health including engine air intake path sensors, manifold pressure sensor and the crankshaft position sensor.
The scheme provided an estimate of immeasurable parameters, redundancy of sensors and effective monitoring. If AIS performance is ensured, the standard stoichiometric ratio can be maintained. On the other hand, a faulty pressure sensor can lead to: Exhaust gases and gas smell, rough idling, poor fuel economy, and hesitation (poor pick up). These faults can be due to bias or drift in sensor outputs. As a preventative measure, early diagnosis of sensor health in addition to the correct selection is necessary.

\section{Physical Models}

For more complex systems, physical descriptions of the system are necessary. The physical models often use computational models to determine the necessary inputs and outputs of the control system rather than relying on closed-loop feedback. The Mean Value Engine Models (MVEMs) approximates the behaviour on a mean value timescale of complex parts. The MVEM can be based on either State Space or Physical models.

M Meza-Aguilar et al [14] proposed an observer-based controller for an internal combustion engine. This estimator is based on sliding mode algorithms, providing a finite time and robust estimation, using only measurements from the velocity of the engine. The engine speed control is complicated. Usually, these controllers are based on MVEMs because it can describe the behaviour of spark ignition (SI) engines. This controller was made using observer design, Engine Adaptive Back-stepping controller design and actuator higher order sliding mode controller design. The states of the SI engine included fuel film flow or mass, crank shaft speed and manifold pressure described by the differential equations; and the control inputs are injected fuel flow rate, spark advance and port and throttle air mass flow.

Fresh charge mass trapped in-cylinder at the end of the gas exchange process is a particularly difficult variable to measure, especially when the throttle position, intake air pressure and engine speed are instantaneously changing. Jingping et al [15] proposed a virtual instrumentation set up consisting of two dynamic pressure transducers to measure pressure fluctuation near the intake and exhaust ports and a descriptor control system. Therefore, the fresh mass and un-swept burnt mass fraction trapped in the cylinder can be calculated throughout the process. The pressure signals are coupled to the numerical solver of a 1-D gas exchange simulation, which is modelled using thermodynamics and hydrodynamics equations (see figure 1). Using the instant flow area at valve locations and the descriptor model, the instantaneous gas mass flowrate through the engine intake and exhaust ports can be calculated. Only the section between the intake and exhaust pressure transducers is modelled which reduced modelling complexity.

Page 3 of 16 
Exhaust system
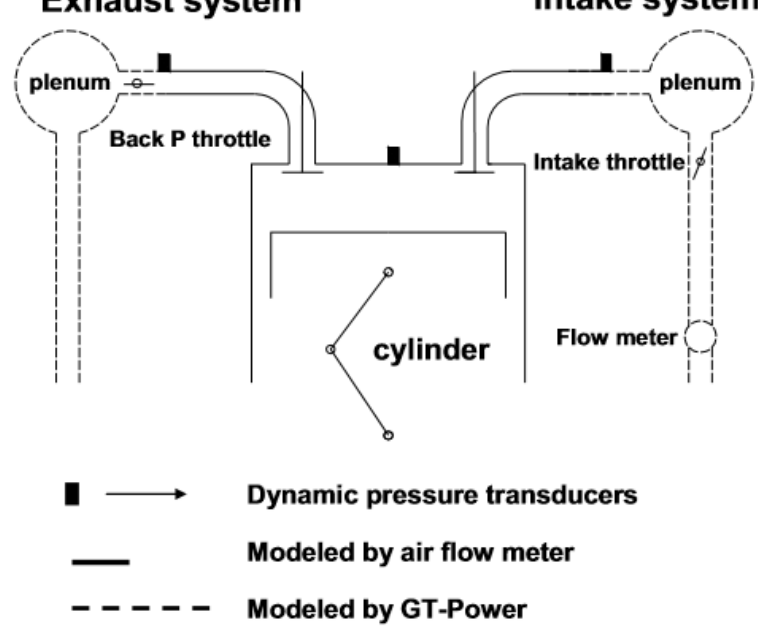

Figure 1. The Virtual Air Flow Meter Set Up proposed by L Jingping et al [15] showing the experimental engine and locations of transducers.

U Vaidya et al [16] proposed an explicit formula for the controllability and observability Gramians as a function of actuator and sensor locations and the advection velocity field. The Gramian based approach is one of the systematic approaches available for optimal placement of actuators and sensors, as their locations are based on where the degree of controllability and observability of the least controllable and observable state is maximized. The problem of actuator and sensor placement in a linear advection PDE is studied, as this approach is particularly useful for the control and detection of temperature and air quality. In order to achieve effective control over shorter duration of time, actuator placement must be in a location where system dynamics expands and spreads over a larger region of the phase space. Moreover, it is shown that the ergodic properties of the vector field are an important factor in actuator and sensor placement for a model based on linear advection.

Additionally, Lee [17] defined controllability Gramians for descriptor based systems, but we will discuss this later in the assessment of control metrics.

Ma et al [18] developed an adaptive valve lift control strategy to improve the intake valve lift repeatability. Electro-pneumatic valve actuators (EPVA) were used as they can control the opening timing, duration and lift of both intake and exhaust valves. The variable valve actuation enables variable valve timing. A descriptor based approach, of linearizing the physics (flow and fluid dynamics, was used to make a control-oriented model. The model reduces computational effort and enables real-time implementation. An adaptive parameter identification algorithm using model reference technique and MIT rule (developed by Astrom et al [19]) were utilized to estimate the two damping coefficients at both opening and closing stages. The identified parameters are then used to construct the feedforward control as part of the closed-loop valve lift Proportional-Integral controller. The MIT rule uses the error between the model and plant outputs to generate the estimated model/plant damping ratio corresponding to the plant/model damping ratio, and is updated at every valve event.
Table 2. Summary of system models

\begin{tabular}{|l|l|l|} 
Authors & System Model & Application \\
\hline Franze [9] & State Space & $\begin{array}{l}\text { Discrete time linear systems } \\
\text { with bounded disturbances }\end{array}$ \\
\hline Munz [10] & State Space & $\begin{array}{l}\text { Sensor and Actuator Placement } \\
- \text { H-2 and H- } \infty\end{array}$ \\
\hline Lee [11] & State Space & Controllability \\
\hline Lee [12] & Modal Domain & $\begin{array}{l}\text { Controllability } \\
\text { Virtual Sensors - Sliding Modes }\end{array}$ \\
Ahmed [13] & Modal Domain & Mean Value Engine Model \\
\hline $\begin{array}{l}\text { Meza- } \\
\text { Aguilar [14] }\end{array}$ & Physical Model & Virtual Air Flow Meter - ICE \\
\hline Jingping [15] & Physical Model & Linear Advection PDE \\
\hline Vaidya [16] & Physical Model & Controllability \\
\hline Lee [17] & Physical Model & Valve Actuator - ICE \\
Ma [18] & Physical Model & \\
\hline
\end{tabular}

Relevant types of system models that can be used to aid sensor and actuator selection for suitable configurations in engines have been identified. State space models can be used for linearized sections of the engine, emphasising the point that dealing with nonlinearities is an important area of work that plays a key role in the selection criteria of sensors. Possible applications of modal domain models are using the resonant frequencies that occur within the manifold combined with the right sensor selection. There is an abundance of literature on physical modelling for automotive systems, yet what is evident is that the impact of selecting a sensor on the performance of the configuration is not discussed in depth. This is one of the reasons for assessing optimisation methods, so that we can determine how applicable these modelling techniques are to design a configuration.

\section{Optimisation based approaches to sensor selection}

There are various methods for optimisation of a system. In the context of sensor and actuator configurations, these include optimal placement and selection. Another approach is to maximise the entropy of the system. Finally, in a software context, the system architecture can be optimised.

\section{Optimal Placement}

The placement methods are based on using the physical location of the transducers to maximise performance based on a certain metric i.e. robustness. An industrial use of actuators is found in active control of helicopter vibrations. In the field of active control of structural response (ACSR), actuator locations have an important influence on the control effect. Q Cheng et al [20] proposed an optimal design method based on the theory of least squares estimation. The air frame structure was modelled as number and location of actuators (design variables), the forces of actuators (restriction condition) and the vibration acceleration responses (dynamic characteristic). The optimization process was then applied to the model. The efficiencies of vibration reduction were observed in three configurations, indicating that the vibration acceleration responses and number of actuators used can be reduced by actuator

Page 4 of 16 
placement. Other systems can also be controlled by optimal actuator placement. A Armaou et al [21] proposed optimal locations of actuators for processes described by parabolic partial differential equations. The particular case that is investigated was transportreaction processes, such as chemical vapour deposition reactors when there is significant time-varying disturbance present. By using modal decomposition for space discretization, spatial distribution of noise and model uncertainty was taken into consideration. The optimization takes care into avoiding actuator locations that excite the higher modes of the system. Figure 2 shows how the modal controllability changes depending on the actuator placement.

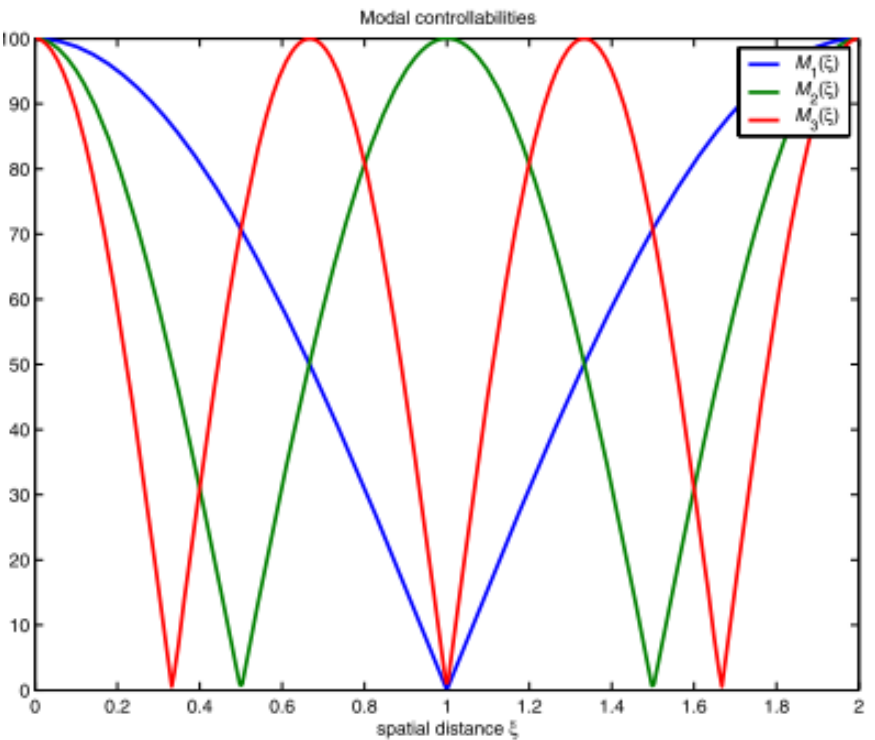

Figure 2. An example of modal controllability of optimal actuator placement by A Armaou et al [21]. The graph represents the percentage controllability of modes one, two and three against the spatial distance of the actuators.

J Yoo et al [22] uses a Gaussian process to estimate a target position against a highly non-linear and noisy Received Signal Strength (RSS). The aim of the work was to find optimal sensor placement for RSS based localization. The Gaussian process is used to construct the localization performance. $\mathrm{N}$ sensors at known locations receive a signal from a target emitting signal strength at an unknown position. The covariance matrix of the noise variance of sensor measurements makes it possible to explicitly analyse configuration. Interestingly, the placement where all sensors have the same distance to the target is not optimal. Likewise, the placement where all sensors are close to the target is not optimal. Therefore, a condition of the optimal angular and distance configuration is that the sensors are apart from each other.

R Skelton et al [23] proposed a method for selecting actuators and sensors in structures, to minimize the instrumental cost. The method consisted of an iterative minimization algorithm to identify the sensor/actuator that requires the least precision from an initial acceptable placement of sensors/actuators. The sensor/actuator with the least precision is removed until a loss of feasibility in the control of the system is noticed. From this approach, the necessary number and type of sensor/actuator, and the location and precision for each sensor/actuator can be identified. The output variance constrained (OVC) problem is to design a dynamic output feedback controller such that the control energy is minimized while each output variance constrain is satisfied. Therefore, economic design is used to determine the location, type and precision of each sensor/actuator as it minimizes the total required precision, while satisfying the system performance constraints. As the price of a component is proportional to its precision; thus the instrumental cost can be expressed in term of the noise covariance matrix.

$X$ Zhao et al [24] investigated engine vibration reduction for aeroplanes using active damping methods and passive systems with squeeze film dampers at bearings. Optimal placement of actuators along the vibration transmission path was assessed against the following measures: robustness of the active system, optimal effectiveness and realizable effectiveness. Nevertheless, active control methods increase the weight of the plane, through the addition of isolating spring and dampers. Other problems with passive techniques include insufficient bandwidth and attenuation to damp the vibration. Zhao et al concluded that the optimal solution is a combination of active and passive.

As previously discussed (see System Models - Descriptor System), Vaidya [16] also investigated optimal actuator and sensor placement, in the context of linear advection PDE. The main contribution was showing that optimal actuator placement depended on a relationship between the system dynamics and phase space.

U Munz et al [10] developed sensor and actuator placement algorithms for linear discrete-time systems based on the closed-loop performance metrics of $\mathrm{H}-2$ and $\mathrm{H}-\infty$ optimization. To test the effectiveness of the algorithm, the optimization process was applied to a power grid, provided by the IEEE 14-bus test system, and the results proved it was highly accurate.

\section{Maximum Entropy}

Maximum entropy uses probability distributions to minimise uncertainty of outputs. R Oseguedo et al [25] addressed the problem of optimal sensor placement for non-destructive testing on aeroplanes by using the maximum entropy approach as a solution. Uncertainty (i.e. state of aircraft) prevents optimization, so symmetry is used to overcome this. The maximum entropy approach calculates probability distributions for different fault locations and sensor placement configurations. This approach involves calculating distributions that are invariant with the symmetries, thus the optimality criterion is also invariant with the symmetries. After several possible sensor locations are found, numerical simulations are run to find the optimal sensor placement. The sensor placement described is for the best relative to all possible reasonable optimality criteria. This is because as the probabilities of faults are unknown, the exact optimality criterion is also unknown. Nonetheless, the shape of the plane must be taken into consideration as this can lead to nonunique optimal placement, because it can be split into perfectly symmetric shapes. When symmetry is only approximate or local, optimal placement is still non-unique. The issue of non-uniqueness is addressed by Group theory which is used to find optimal families of sets e.g. groups of rotations. Every set from the optimal family consists of one or several orbits of subgroups of the original transformation group.

\section{Systems Engineering}

Systems engineering is applicable to the optimization of the control architecture on a higher level. R Kudikala et al [26] used multicriteria optimization for system architecture design in the case of an aero engine health management (EHM) system. Due to the large and discontinuous design search space, there are many qualitative and quantitative criteria that must be considered. However, an evolutionary multi-objective genetic algorithm (MOGA) and a progressive preference articulation technique, is used to solve the optimization problem. Therefore, it is necessary to identify the following key components: a model of the system, its decision

Page 5 of 16 
variables, the objective functions to be optimized and system constraints. For designing the EHM functional architecture for the aero engines, all primary EHM functional requirements and stake holder requirements are captured using requirements analysis and flow-down techniques, and represented as EHM system use cases. The EHM system primary use cases are decomposed into several EHM functions and functional operations (OPs). 'Criticality', 'Immediacy', 'Coupling', 'Security', 'IP sensitivity' and 'Flexibility', are considered as six individual objective functions to be minimized in the optimization process. Furthermore, the process of introducing, incorporating and modifying designer preferences in an interactive and progressive way at any time during the optimization search process; is a key feature for multi-criteria decision making (MCDM). The paper identifies that it is not possible to fully satisfy all attributes for the EHM system, while observing the given constraints, thereby highlighting the value of a multi-criteria approach.

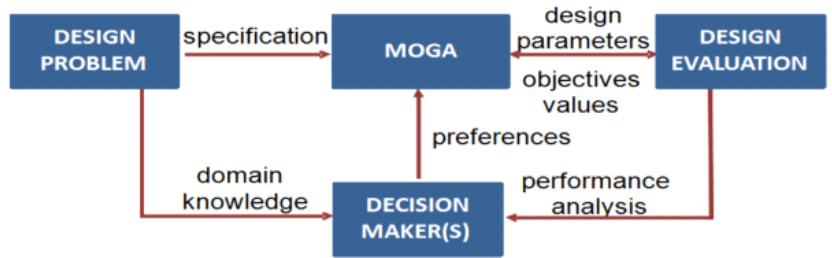

Figure 3. The Interactive Multi-Criteria Optimization Design Framework proposed by Kudikala [26].

\section{Optimal Selection}

Another industry that uses sensor and actuator filters are autonomous vehicles. C Moreno et al [27] proposed an approach, based on robust controllers accounting for model uncertainty, in sensor and actuator selection on aircraft (in particular, aero-servo-elastic systems). Control design-independent methods are based on measures of controllability and observability of the plant. Therefore, robust fullinformation controllers are designed for different actuator configurations, and their performance is compared to obtain an optimal selection. This is followed by designing robust full-control controllers for different sensor configurations and using similar techniques to obtain an optimal selection. The general control synthesis configuration for uncertain systems helps define the robust control problem; which is to find a stabilizing controller that minimizes the $\mathrm{H}-\infty$ norm of the closed-loop transfer function while maximizing the robustness against the uncertainty. After physical testing on a small unmanned autonomous vehicle, it was observed that the sensor selection has a greater effect on the robustness performance than the selection of actuators.

Huber et al [28] described a methodology for the selection of sensors for the diagnosis of faults within internal combustion engines. Faults were represented using parameter estimation to add states to the plant model. Redundant sensors are used for state estimation to identify faults and exclude faulty sensors. Although this can reduce system performance, it enables fault tolerant operation. Using a non-linear MVEM, an Extended Kalman Filter (EKF) is applied to perform the state and parameter estimation. The aim of the selection methodology is for a given set of faults, which are considered critical for operation, the lowest number of sensors are chosen to guarantee that the considered faults are detectable and can be isolated from others. As defined in the paper, 'the optimal sensor configuration maximizes the minimal eigenvalue of the observability Gramian by the choice of sensor configuration and the related output matrix'.
Table 3. Summary of optimization methods of sensor and actuator selection.

\begin{tabular}{|l|l|l|}
\hline Authors & Optimization Method & Application \\
\hline Cheng [20] & Placement & $\begin{array}{l}\text { Active Control - Helicopter } \\
\text { Vibrations }\end{array}$ \\
\hline Armaou [21] & Placement & $\begin{array}{l}\text { Modal Controllability - Optimal } \\
\text { Actuator Placement }\end{array}$ \\
\hline Yoo [22] & Placement & $\begin{array}{l}\text { Received Signal Strength based } \\
\text { localization }\end{array}$ \\
\hline Skelton [23] & Placement & Economic Design \\
\hline Zhao [24] & Placement & Actuator - Aircraft Engines \\
\hline Vaidya [16] & Placement & Sensor and Actuator \\
\hline Munz [10] & Placement & H-2 and H- $\infty$ \\
\hline Oseguedo [25] & Maximum-Entropy & Aerospace Non-Destructive Testing \\
\hline Kudikala [26] & Systems Engineering & Real World System Design \\
\hline Moreno [27] & Selection & Autonomous Vehicles \\
\hline Huber [28] & Selection & State and Parameter Estimation \\
\hline
\end{tabular}

This section is crucial to understanding the limitations of selection criteria. It shows the types of optimization methods that can be used for sensor selection. The literature is divided into optimization based on placement, maximum entropy, systems engineering and selection methods. The placement methods are based on using the physical location of the sensors and actuators to maximise performance based on a certain metric i.e. robustness. Maximum entropy uses probability distributions to minimise uncertainty of outputs. Systems engineering is the optimization of the control architecture on a higher level. Also, the selection methods are based on system state estimation. This section raises the issue that approach to building configuration can have a significant impact on trade-offs between performance and cost. Therefore, understanding how much information contained within the system is highly valuable and maximum entropy for optimisation can be an appropriate method to determine this. However, one of the limitations of using entropy is that it does not explicitly describe the usefulness of the information. As the engineer designing the system it must be considered whether a top down approach to building the configuration by assessing the high-level control architecture that oversees the whole system needs to be optimised or whether to focus on individual sections of the engine and build the configuration from the bottom up.

\section{Assessment of Control Metrics}

Many of the papers reviewed perform controllability analysis on their respective system. Control metrics are defined to assess performance quality and improve optimization in different areas. These are considered to be hard metrics, which are categorized into: Degrees of Freedom, Closed-loop, Open-Loop and Miscellaneous. 


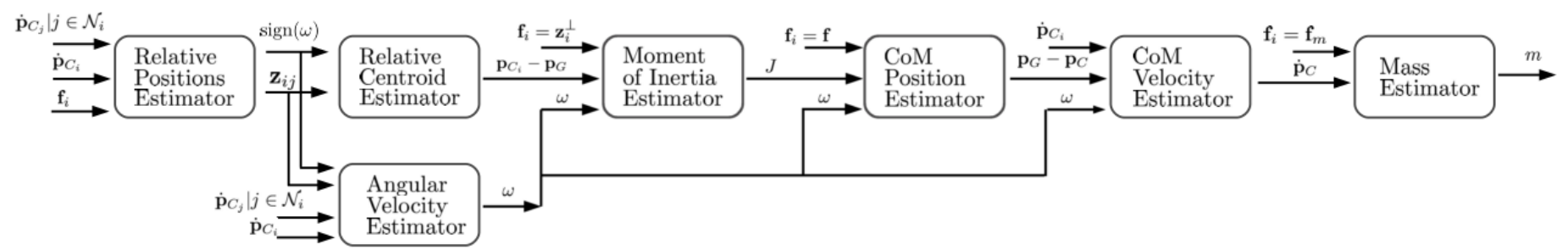

Figure 4. An example of model inversion to estimate variables proposed by Franchi et al [32].

\section{Closed Loop Performance}

\section{$H-2$ and $H-\infty$}

$\mathrm{H}-2$ and $\mathrm{H}-\infty$ optimization was used for optimal transducer placement by U Munz et al [10]. An observer was designed for sensor placement that minimizes the H-2 norm of the error dynamics and the number of sensors simultaneously. Similarly, a state feedback controller is designed for actuator placement that minimizes the $\mathrm{H}-\infty$ norm of the closed-loop system and the number of actuators simultaneously. Due to the fact that algorithms are proposed for both $\mathrm{H}-2$ optimal sensor and $\mathrm{H}-\infty$ placement, the proposed method can also be applied to controller and observer design and continuous-time systems. As previously outlined, C Moreno et al [27] proposed an approach for sensor and actuator selection on aircraft. This was also done by developing a robust controller based on $\mathrm{H}-2$ and $\mathrm{H}-\infty$ techniques.

\section{Covariance}

Another closed loop control metric considered is the covariance between variables. This is particularly important when considering uncertainty within the system, as discussed within the maximum entropy method of optimisation. A Bertrand et al [29] described a distributed adaptive (time-recursive) algorithm to estimate and track the eigenvectors corresponding to the Q largest or smallest eigenvalues of the global sensor signal covariance matrix in a wireless sensor network. The algorithm proposed converges to the desired eigenvectors without explicitly constructing the global covariance matrix that actually defines them. This means that the desired eigenvectors are found without the need to centralize all the raw sensor observations. Data fusion is used to increase the accuracy of the measurement being performed and to overcome reliability issues in sensors and uncertainty in output, making the system more robust. Sequeira et al [30] used the robust estimation of a covariance matrix to express uncertainty when fusing information from multiple sensors. Additionally, the differences between estimators using explicit measurements from the sensors involved and estimators using only covariance estimates from the sensor models and navigation systems are also investigated. The paper focused on the estimation of a covariance matrix, using an Orthogonal Gnanadesikan-Kettenring (OGK) estimator, for a single landmark that has been observed by two different sensors. Analysis showed that there are regions of the spectrum of the covariance matrix where each of the estimators outperforms the other; implying that a hybrid of the two estimators can provide the best results.

\section{Robust Stability}

Traditional focus has been on developing a controller to stabilize the dynamic system when system parameters perturb in certain bounded range. Alternatively, X Li et al [31] investigates the robust inverse

Page 7 of 16 problem for linear systems, where they attempt to find a stable system parameters' perturbation space for any given stable controller. Each system parameter must be considered as each has different effects on robust stability of the dynamic system or process. The inverse theorem states that any given linear closed-loop control system is asymptotically stable and the controller can stabilize dynamic systems or processes. Then processes must at least have a parameter perturbation space or robust stability domain and the controller can still stabilize the control system when system parameters of processes perturb in the same space. To demonstrate the theorem, a PID Controller is used to stabilize a nominal perturbation process.

\section{Estimation and Manipulation Theory}

Control systems controllability is integral with its ability to estimate variables using sensors and manipulate the plant using actuators. A good example of this is proposed by A Franchi et al [32]. Their paper describes a distributed strategy for the estimation of the kinematic and inertial parameters of an unknown body manipulated by a team of autonomous ground vehicles (mobile robots). Kinematics and dynamics arguments are used to estimate the relative positions of the contact points on a payload. The inertial parameters including mass, relative position of the centre of mass and moment of inertia are estimated using distributed estimation filters. The inertial parameters estimation is defined through an algorithm (figure 4), which is able to define constant parameters mass and inertia and the time varying relative position vector; whilst only controlling local force, using local information regarding speed and communicating with neighbours. The inversion used is implicit not explicit. In terms of control architecture it demonstrates how many signal flows within the system and how system structure is an important factor when considering the sensors and actuators selected.

Table 3. Summary of closed loop performance metrics

\begin{tabular}{|l|l|l|}
\hline Authors & Metric & $\begin{array}{l}\text { Applications } \\
\text { Sen/Act Placement - Linear discrete- } \\
\text { time }\end{array}$ \\
\hline Munz [10] & H-2 and H- $\infty$ & Autonomous Vehicles \\
\hline Bertrand [29] & Covariance & $\begin{array}{l}\text { Eigenvectors of matrix in wireless } \\
\text { sensor network }\end{array}$ \\
\hline Sequeira [30] & Covariance & Data fusion from multiple sensors \\
\hline Li [31] & Robust Stability & Robust Inverse for Linear Systems \\
\hline Franchi [32] & $\begin{array}{l}\text { Estimation and } \\
\text { Manipulation Theory }\end{array}$ & Autonomous Ground Vehicles \\
\hline
\end{tabular}

\section{Open Loop Performance}

Open loop control metrics are concerned with the maximum entropy distribution which is constrained by the available data; which is the 
joint distribution of the random variables that best describes current knowledge with no further assumptions. D Cochran et al [33] investigated multiple-channel detection in the context of a sensor network where raw data are shared only by nodes that have a common edge in the network graph. This is an interesting method if we consider the sensors on an engine to be a network made up of the signals that are contained within the control architecture. They use a maximum-entropy technique to formulate surrogate values for missing measurements corresponding to pairs of nodes that do not share an edge in the network graph. Their approach eliminates the need for a 'fusion-centre' where data is collected and processed at a central point in the network. Using this approach, data can be processed at nodes, locally. The technique was applied to a small network with limited connectivity. The performance of the connections was then reduced and the effectiveness of the technique was observed. Performance degradation of the network was modest, but to observe the true quality of the approach, it needs to be applied to a larger network.

S Rajasegarar et al [34] proposed a framework combining Bayesian compressive sensing and a robust Bayesian maximum entropy (BME) based spatio-temporal estimation technique. The paper investigates large scale sensing, which requires a large numbers of sensors deployed in a region for accurate and high resolution spatio-temporal measurements and estimation. Factors that contribute to successful large scale sensing include high capacity, high precision, expensive and low capacity, low precision, cheap sensors in the monitored region. However, resource constraints and the availability of highcapacity sensors are a challenge to achieving highly accurate estimations.

One approach to successfully achieving large scale sensing is to use a compressive sensor, which exploits low sampling rate and is capable of processing at the node. Another method to improve large scale sensing is to use BME to estimate the value of the observed phenomena at arbitrary locations. The approach combines two prime knowledge bases. Firstly, a general knowledge base, such as from physical laws, summary statistics and scientific theories. Secondly, a case specific knowledge base obtained through experience with specific situations. The specific knowledge base accounts for both hard data (e.g. exactly measured values), and soft data; which is data with uncertainty due to factors including: inexact knowledge, experience, intuition, low precision sensor outputs. Results of simulation on a real wireless sensor network showed a trade-off between spatio-temporal estimation accuracy and the communication overhead in the network.

Table 4. Summary of open loop performance metrics - maximum entropy.

\begin{tabular}{|l|l|}
\hline Authors & Maximum Entropy \\
\hline Cochran [33] & Multiple Channel detection of sensor network \\
\hline Rajasegarar [34] & Spatio-Temporal Estimation \\
\hline
\end{tabular}

\section{Degrees of Freedom}

H Lee et al [11] defined a measure which can consider both controllability and observability simultaneously. This is the degree of compensation capability (DOCC) and degree of output noise sensitivity (DONS). DONS is a measure that represents the effect of output noise to control input and it can be represented by the trace of the inverse of controllability and observability Gramian. DOCC is a sum of three terms including DONS, degree of controllability and degree of observability. The measure involves minimizing energy transfer between sensor and noise, which is a measure of disturbance Page 8 of 16 rejection. So DONS contains physical meanings of both controllability and observability. However, it does not mean the locations of actuators and sensors with the optimal value of DOC and DOO are same as that with the optimal value of DONS. Later, Lee et al [12] also defined controllability and observability Gramians in the modal domain. Then by using the definition of modal DONS, the locations of actuators and sensors to control of each individual mode can be determined by considering the effect of output noise to input. Finally, H Lee et al [17] defined measures for controllability in a descriptor system. The degree of controllability (DOC) for descriptor systems is defined as the minimum input energy to change the states. To improve the controllability of the systems, the DOC should be minimized by actuator placement. The results of the proposed measure show that the input energy to change the state of the Gramian is only affected by the final condition and of slow systems which means that the fast systems does not affect the input energy. Vaidya [16] also defines metrics for controllability and observability in descriptor systems. Gramians are defined for optimal actuator and sensor placement in linear advection PDEs.

Furthermore, J Huber et al [28] defined another metric in the context of fault tolerance. This is the degree of fault observability, which is adapted from controllability. Huber also defined the Fault Observability Index, which is 'the ratio of maximum to minimum eigenvalue of the fault observability Gramian'. A lower index is desirable because the measure is invariant to similarity transforms.

Table 5. Summary of degrees of freedom metrics

\begin{tabular}{|l|l|}
\hline $\begin{array}{l}\text { Authors } \\
\text { Lee }\end{array}$ & $\begin{array}{l}\text { Degrees of Freedom } \\
\text { Compensation Capability }\end{array}$ \\
\hline (Linear) [11] & Output Noise Sensitivity \\
\hline (Modal) [12] & Output Noise Sensitivity \\
\hline (Descriptor) $[17]$ & Controllability \\
\hline Vaidya [16] & Controllability \\
\hline Vaidya [16] & Observability \\
\hline Huber [28] & Fault Observability \\
\hline
\end{tabular}

\section{Fault Tolerance}

In the case of sensor and actuator selection criteria it is important to consider the fault tolerance of the system. J Dai et al [35] addressed fault-tolerance of multi-agent systems. The behaviour of each agent in the system is described as a local automaton and the system behaviour as the composition of automatons. Sensor failures occurring in individual agents are defined as loss of observability of a local event of a failed agent, and actuator failures are modelled as a total loss of an event from an agent. Sensor failures are modelled by permanent loss of local observability and controllability (whenever it is locally controllable) of certain local events; and actuator failures are modelled as a loss of certain events from the corresponding agent's local event sets. A learning-based algorithm is proposed to resynthesize appropriate local supervisors when sensor failure occurs, and target for the necessary and sufficient conditions under which the global specifications are maintained under actuator failures. G Franzè et al [9] proposed a novel actuator fault tolerant control strategy for constrained discrete time linear systems subject to bounded disturbances. The fault tolerance scheme consisted of three modules. The first module was a bank of estimators, each one 
associated with healthy and faulty model configurations. The second module was a logic mechanism for identifying healthy-to-faulty and faulty-to-healthy transitions, which takes into account correctness and admissibility. The third module was an estimate based control reconfiguration unit. Initially, the scheme described different plant configurations through a switching paradigm and sequences of approximations of controllable sets. A switching logic is then used to determine the current plant configuration on the basis of the state estimate provided by the observers. There are two critical points in the strategy, how to distinguish between configurations and how to ensure a correct model configuration switching; whilst closed-loop stability and constraint fulfilment are preserved.

$\mathrm{J}$ Machado et al [36] proposed a new method for the calculation of the fractional expressions in the presence of sensor redundancy and noise. The algorithm used is tuned and optimized through genetic algorithms. A Genetic Algorithm (GA) is a computational technique to find approximate solutions in optimization and search problems. GAs are simulated through a population of candidates that evolve computationally towards the better solution. GA take advantage of signal characteristics and sensor redundancy. The redundancy means that different signals can be processed through alternative algorithms and still lead to the same state variable. However, implementation issues such as sensor noise must be considered when creating a system with redundancy. The proposed scheme was successful in adapting to different fractional expressions for particular sensors and noise.

Table 6. Summary of examples of fault tolerance.

\begin{tabular}{|l|l|}
\hline $\begin{array}{l}\text { Authors } \\
\text { Dai [35] }\end{array}$ & Fault Tolerance \\
\hline Franze [9] & Discri-Agent Systems \\
\hline Machado [36] & Fractional Expressions using sensor redundancy \\
\hline
\end{tabular}

This section has outlined the types of control metrics that can be used to assess the performance of a model. Whether the system is closed loop or open loop we have metrics that can be used to determine its quality. The literature has showed that $\mathrm{H}-2, \mathrm{H}-\infty$, covariance, robust stability and estimation/manipulation theory can be used to quantitatively assess the performance of a control system. The usefulness of these assessment metrics is limited but used in combination with one another they can achieve desired trade-off of the control architecture. The applications of these metrics showed that an alternative method to view the control architecture is to consider how signals flow within the system and how system structure is an important factor when considering the sensors and actuators selected. As previously stated, this is an interesting method if we consider the sensors on an engine to be a network made up of the signals that are contained within the control architecture. Sensors could then be selected to make a robust information centric configuration, which could make full use of the information available within the architecture.

\section{Historical Research}

This section is based on literature which is at least 5 years old, with some going back to the 1980s. This offers an opportunity to assess topics that at one point were of great interest to the field of optimal configuration.

Page 9 of 16

\section{Applications}

Hammerschimdt et al [37] assessed the role of sensors for an ECU in automotive applications. They state that there are two types of sensors: offshore and local. Offshore sensors must sense the physical values at a location away from the ECU, whereas the local sensors do not have to be placed near the physical value. The types are divided by the need of power and communication between the ECU and sensor. This allows the designer to optimize the choice of sensors depending on functionality, safety, reliability, durability and availability. Thus other metrics can be modelled in the design stage. McKelvey et al [38] used sensors to gain a better understanding of the combustion process for optimization. By using a crankshaft mounted torque sensor and a signal processing technique, closed loop ignition timing control is achieved. Estimation of combustion phasing for each cylinder while compensating for torsional was carried out. The result was a reduction in fuel consumption of the 5 cylinder in line spark ignition engine. Balau et al [39] successfully modelled an electromagnetic valve actuator for clutch control in an automatic transmission. It was based on a linearized input-output model, with a suitable transfer function for Simulink, as well as a state space model. Furthermore, Aono et al [40] proposed a signal processing algorithm for compensating for the back flow effect in intake air mass measurement in internal combustion engines. This used frequency characteristics of a signal from an air flow sensor. This technique demonstrates how signal processing can be performed on an engine to find out new information and how correlation between signals can be exploited.

Table 7. Summary of historical applications within industry.

\begin{tabular}{|l|l|}
\hline Authors & Applications \\
\hline Hammerschidmt [37] & Sensor interfaces \\
\hline McKelvey [38] & Crankshaft mounted torque sensor \\
\hline Balau [39] & Electromagnetic valve actuator for automatic transmission \\
\hline Aono [40] & Signal Processing technique for back flow effect \\
\hline
\end{tabular}

\section{Control metrics}

\section{Robust Stability}

Van de Wal has been identified as a relevant author in the field of input/output sensor selection methods. This is an early paper which covers the work of the selection methods review paper (which we will discuss later), Philips et al [41] aimed to eliminate actuator/sensor combinations for which no controller exists that achieves a specified level of robust performance. Additionally, complete controller synthesis is avoided by using necessary conditions for robust performance, but this highlights one of the limitations of the method; stabilization for certain combinations is not guaranteed. The research uses structured singular value theory and states that the effectiveness of the combination is strongly affected by the choice of design filter.

Rotea et al [42] identified the relations between two types of stability: quadratic and robust (formal definitions are provided). The systems assessed were uncertain systems with structured uncertainty due to both real and complex parameter variations. One of the learning outcomes is that for systems containing at least two uncertain blocks quadratic stability for real perturbations does not imply quadratic stability for complex perturbations. This shows that there are 
different types of stability depending on the structure of the uncertainty of the system.

\section{Right Half Plane Zeros}

Pandolfi et al [43] observed the effects of right half plane (rhp) zeros on sensitivity reduction. The paper is very short in length but explains the effects of the location of unstable zeros, multiple zeros and transmission delays. Also, control feedback design is considered including stability, sensitivity reduction and robustness. Another paper that looks at the effects of right half plane zeros was produced by Cheng et al [44]. The focus was on the limitations of the closedloop transfer function of any stable feedback system due to rhp zeros. However, this research is more relevant to electronics, than being applicable to an engine.

\section{Closed Loop}

As discussed in optimal placement methods, Skelton [23] proposed an Economic design approach to actuator/selection. Norris et al [45] published a paper on actuator selection when noise is present. This is based on Closed-Loop Input/Output Cost Analysis (CIOCA). Due to the methods associated with the technique, it was demonstrated that performance may be degraded when a transducer is noise free.

\section{Open Loop}

Li et al [46] proposed research on multi-sensor correlation analysis for control systems in coal mines. It used a fast algorithm that calculates the combinations of correlative sensors. The paper shows the use of condition number in systems with a large number of sensors. Similarly to the previous paper mentioned, Welsh et al [47] identified an application of the condition number. In this case, the focus is on wide-band system identification, as parameters of a system with a large dynamic range are difficult to compute for a least squares type algorithm. A condition number of the least squares problem, which is independent of the frequency range for a particular class of models; is used as a bound for output errors of the system.

Table 8. Summary of historical control metrics.

\begin{tabular}{|l|l|} 
Authors & Control Metrics \\
\hline Philips [41] & Robust stability used in selection methods \\
\hline Rotea [42] & Quadratic and robust stability \\
\hline Pandolfi [43] & Effects of right half plane zeros on sensitivity reduction \\
\hline $\begin{array}{l}\text { Cheng [44] } \\
\text { Norris [45] }\end{array}$ & $\begin{array}{l}\text { Effects of right half plane zeros } \\
\text { Closed-Loop Input/Output Cost Analysis }\end{array}$ \\
\hline Li [46] & Condition number \\
\hline Welsh [47] & Condition number for wide-band system \\
\hline
\end{tabular}

\section{Fault Tolerance}

An approach to fault tolerant detection and identification was proposed by Seron et al [48], using an invariant set combined with a virtual actuator to controller reconfiguration. It is based on the separation of invariant sets that characterise healthy system operation from those that describe faulty operation. The benefit of this scheme is that any existing nominal controller that satisfies the desired specifications for the plant can be kept in the loop at all times.
Moreover, the virtual actuator is used to preserve closed-loop properties under fault such as stability and set-point tracking. It was concluded that the faults have negligible effect on the plant states, which track the desired reference almost perfectly. The use of virtual actuators to create a fault tolerant control scheme is an alternative selection. Furthermore, optimal design of fault tolerant sensor networks was reviewed by Hoblos et al [49]. The paper covered the design of sensor networks such that the observability of the variables for the process control remains satisfied under faults. Definitions are provided on Pseudo-minimal and minimal sensor sets. These sets are organized into an oriented graph which contains all the possible reconfiguration paths for which those variables remain observable. The overall objective was to improve the robustness of the observability property. A highly relevant paper was found during the search of the literature review that described a method of assessing sensor quality in a system. Chen et al [50] proposed a novel real-time fault compensation method, which uses state estimation and compensation techniques (state feedback), that allow the sensor system to perform robust measurements even when sensor outputs are noisy and drifting. It identified that geometric redundancy is an important design aspect for fault tolerance. The method was also capable of compensating sensor drifts without affecting the sensor accuracy. Virtual actuators were also used by Richter [51] for the control reconfiguration of a thermo-fluid process. As outlined previously, it hides the fault from the controller, allowing the nominal controller to remain in the loop. The reconfiguration goals are: Fault hiding goal, stabilisation goal, equilibrium recovery goal, trajectory recovery goal. It was concluded that the linear virtual actuator is able to reconfigure processes with nonlinear behaviour.

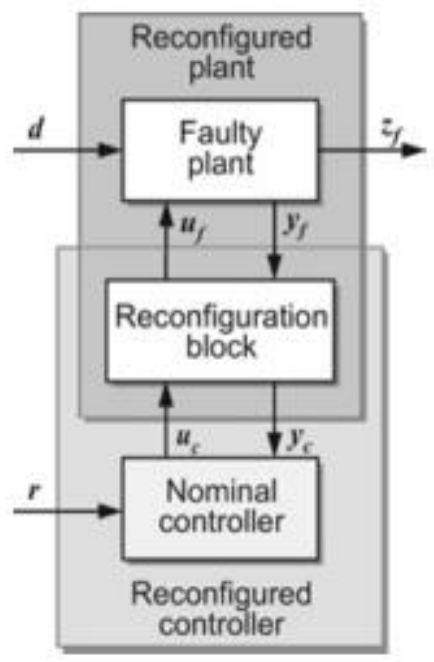

Figure 5. Reconfigured loop with reconfiguration block by Richter et al [51]. This is an example of designing virtual actuators using loop shifting methods.

Table 9. Summary of historical examples of fault tolerance.

\begin{tabular}{|l|l|}
\hline Authors & Fault Tolerance \\
\hline Seron [48] & $\begin{array}{l}\text { Invariant set combined with a virtual actuator to controller } \\
\text { reconfiguration }\end{array}$ \\
\hline Hoblos [49] & Optimal design of fault tolerant sensor networks \\
Chen [50] & Novel real-time fault compensation method \\
\hline Richter [51] & Control reconfiguration of a thermo-fluid process \\
\hline
\end{tabular}

Page 10 of 16 


\section{Optimization}

\section{Placement}

Actuator and sensor placement is fundamental to selection criteria and Chevrel et al [52] proposed a methodology using convex optimization tools to determine the optimal placement. The controllability and observability of a system are dependent on the transducer locations. There are four optimization problems which are defined in the paper: sensor placement, sensor placement in worst case, actuator placement and actuator and sensor placement. These are equivalent to a linear objective optimization problem under constraints, which are solvable using the complex tools. Antoniades et al [53] proposed an integrated optimal actuator placement and robust control of uncertain transport reaction processes. The results were successfully applied to a diffusion-reaction process with uncertainty. Similar methods were found in the optimization review of recent literature.

CanXing et al [54] reviewed optimal sensor placement for health monitoring. Cause-Effect analysis methods were used including: Fault tree method, Petri-Net method, and Graph theory. Optimization was also split between component and system level. Although it is a short review paper, the general optimal approaches for sensor placement are presented. Finally, it is identified that Demetriou and Armaou have produced research on optimal placement that is particularly relevant to this field. An earlier conference paper [55] of early work was reviewed, which also used a spatial H-2 norm.

\section{Selection}

Chmielewski and Peng presented two papers on Covariance based hardware selection the first is on globally optimal actuator selection [56]. Through a linear matrix inequality (LMI) based transformation the original problem is converted into a mixed integer convex program (MICP). The MICP is capable of calculating globally optimal solutions to the covariance based actuator selection problem. The second paper [57] is based on the equivalence results for the sensor, actuator, and simultaneous selection problems. It is concluded that 'significant advantages can be found in the suboptimal controller version of the actuator selection problem. In particular, the suboptimal version can be used to find global solutions to the previously intractable optimal controller formulation'. The advantages of using suboptimal actuator selection strategies are also discussed by Vanbeveren et al [58]. They provide a definition of the cost function which is 'the integral of a weighted combination of the achieved accuracy on the state of the system and the control energy'. The control energy term is dependent on the selected actuator and the magnitude of the applied control. They use a sub-optimal 'ForwardBackward' Algorithm which is used when certain actuators can be rejected a priori.

\section{Systems Engineering}

One of the older papers of the literature review was produced by Lynch et al [59], who proposed an object oriented intelligent control architecture. The objects, which contain data, functions and inherited knowledge, represent: sensors, a sensor manager and a controller. The intelligence is embedded within each object, allowing for the construction of efficient systems. The paper is useful because it critically assesses the use of architectures in control systems.

Another method of creating control architecture is proposed by Voulgaris et al [60]. Certain optimal control problems are considered, with constraints on the processing of the measurement data. The two types of problems are based on whether the structure of the plant that Page 11 of 16 relates controls to measurements has the same structure in the feedthrough term as the one restricted by the observation pattern. Loop shifting ideas similar to those found in fault tolerance (figure 5) are used when the structure is not the same. The approach is based on one step delay observation sharing pattern.

Clarhaut et al [61] extensively described optimal control architecture design. A complete design methodology for dependable systems is outlined. Hierarchical functional decomposition of the system is carried out for efficiency. This divides the architecture into functional, equipment and operational. It is at this stage a dependability level is either static or dynamic. Failure relationships between functions and dependability are calculated through multifault tree representation. This paper identifies that we must also review soft metrics for a complete optimization of the system. Hammerschmidt et al [37] outlined useful soft metrics in the selection of control architecture. Other metrics include: Design Complexity (Effort of Controller Design), Uncertainty, Skill Based, Customer Experience, Connectedness, and Qualitative metrics - existence of sign reversal (loop gain change). Systems engineering is where the majority of this literature will be found. Pugh matrix analysis of these metrics would be a useful indicator for sensor selection criteria. Freudenberg et al [62] investigated design trade-offs in feedback systems. Physical realizability is emphasised as the origin of the trade-offs assessed in the paper. This is different to trade-offs between performance and robustness. The paper also identifies limitations on the sensitivity function to open rhp zeros and unstable poles.

Bushnell et al [63] designed an expert systems solution (SYSMON) to 'Automate the function of a Junior Control Engineer' in an early paper. Object oriented concepts such as inheritance, encapsulation and polymorphism were used in the design of architecture for transmissions system monitoring. The modules used in the system included data acquisition, on-line analysis, predictive applications, human computer interface and other specific expert system modules. Chan et al [64] developed object oriented architecture of control system for agile manufacturing cells. There are three modes to manufacturing cell control: centralized, hierarchal and heterarchical. The objects are divided into: control objects, resource objects (supervisor, functional component and driver) and information objects. The architecture improves the controller design because the system is simplified, communication is now message based and objects are reusable.

Table 10. Summary of historical optimization methods.

\begin{tabular}{|l|l|}
\hline Authors & Optimization \\
\hline Chevrel [52] & Placement using convex optimization tools \\
\hline Antoniades [53] & Optimal actuator placement for transport reaction processes \\
\hline CanXing [54] & Optimal sensor placement for health monitoring \\
\hline Demetriou [55] & Optimal actuator placement \\
\hline $\begin{array}{l}\text { Chmielewski [56], } \\
\text { Peng [57] }\end{array}$ & Globally optimal actuator selection \\
\hline Vanbeveren [58] & Suboptimal actuator selection strategies \\
\hline Lynch [59] & Object Oriented Intelligent Control Architecture \\
\hline Voulgaris [60] & $\begin{array}{l}\text { Optimal control of systems with delayed observation sharing } \\
\text { patterns }\end{array}$ \\
\hline Clarhaut [61] & Optimal Design of Dependable Control System Architectures \\
\hline
\end{tabular}




\begin{tabular}{|l|l|}
\hline Freudenberg [62] & Design trade-offs in feedback systems \\
\hline Bushnell [63] & Application of object oriented techniques \\
\hline Chan [64] & Object-oriented architecture of control system \\
\hline
\end{tabular}

\section{System Models}

A different form of system modelling proposed is the use of a neural network, which is used by Papadimitropoulos et al [65] for fault detection in mechanical systems, performing linear motion with friction. The neural fault detection methodology is assessed by robustness and sensitivity properties.

Sarrate et al [66] proposed an algorithm for model-based fault detection and isolation sensor placement based on formulating a mixed integer optimization problem. Constraints are used to create the optimization problem based on a set of analytical redundancy relations and a fault signature matrix, which used isolability and detectability.

Alternatively, plant based modelling was used by Zhang et al [67] for the robust control of interval plants. Interval ranges of model parameters are used to determine the closed-loop control actions of the system. The paper also states that interval models are useful descriptions for uncertain dynamic processes.

Table 11. Summary of historical system models.

\begin{tabular}{|l|l|}
\hline Authors & System Models \\
\hline Papadimitropoulos [65] & Neural Network \\
\hline Sarrate [66] & Model-based fault detection and isolation \\
\hline Zhang [67] & Plant based modelling \\
\hline
\end{tabular}

This section identified some non-technical metrics (i.e. dependability and reliability) that can be used for optimization. Systems engineering is the most relevant field that can be used to find literature on this form of optimization and as stated a Pugh matrix analysis of these metrics would be a useful indicator for sensor selection criteria. These are important criteria for the selection of sensor and actuator. This literature also reiterates the need an understanding between the optimisation at the different levels of the system. It is shown that optimisation can be carried out on multiple levels including high level overall system (i.e. engine health management) and low level (i.e. sensor placement on the air system control).

\section{Selection Methods Review Paper}

It should be stated that a review paper by Van de Wal [68] was studied. The paper is titled 'A review of methods for input/output (IO) selection'. It gives a clear outline of control system design based on 8 desirable properties of IO selection methods: Well-founded, Efficient, Effective, Generally Applicable, Rigorous, Quantitative, Controller Independent, and Direct. A Pugh matrix is used to assess the classes of IO selection methods that were review in the paper. The paper outlined many open loop and closed loop performance metrics that are referred to as IO selection criterion.

\section{Patent Search of Sensor Selection Methods}

The results of the patent search demonstrated that only patents on control strategies and sensors were found. Nothing was found on selection methods; however, the focus was on automotive patents. We did not look into electronics, control or computing fields, where potentially relevant patents can be found here.

The subcategory F02D 41/1401 is especially relevant:

- Electrical control of supply of combustible mixture or its constituents (F02D43/00 takes precedence)

- Circuit arrangements for generating control signals

- Introducing closed-loop corrections

- Characterised by the control or regulation method (F02D41/1473, F02D41/1477 take precedence)

- Controller structures or design

The following patents have been identified to be relevant, because they apply to specific solutions (and certainly further such patents exist):

- $\quad$ KR20150122935 (A) - Exhaust processing device control method for vehicle [69]

- US2013197779 (A1) - Setpoint Bank Control Architecture [70]

- US2016215749 (A1) - Control device of internal combustion engine [71]

- $\quad$ KR20160056822 (A) - Method for processing a signal of combustion chamber pressure sensor [72]

- US2016237933 (A1) - Method and apparatus for controlling a reciprocating-piston engine having several cylinders [73]

- JPS5895214 (A) - Signal processing method for hot-wire flow rate sensor [74]

- $\quad$ EP1413728 (A2) - Controller and method for controlling a NOX-sensor arranged in an exhaust gas channel of an internal combustion engine [75]

\section{Conclusions}

Having extensively and systematically reviewed the literature, we have provided an overview of the current state of selection criteria for suitable sensor and actuator configurations for internal combustion engines. System Modelling, Optimisation and Control Metrics are the three key areas of study for this topic. The patent search found many patents for specific sensors and control schemes, but there is no evidence of a systematic approach to sensor and actuator selection. An engine is an ideal opportunity to apply these methods because success in each of the key areas have been found in many alternative industries across engineering. The review aimed to answer the following questions to assess these selection criteria:

1. Which industries use sensor/actuator selection filters? Examples of industries using selection filters include Exoskeleton, Process Control, UAV, Automotive, and Aeronautical.

2. Which methods are used in sensor/actuator selection? No consensus is found on a particular selection method, but development of robust full information/control controllers has been suggested.

3. What are the specific challenges of engine control? To be fully answered the question needs to be divided into engine subsystems. 
4. How much data can you get through sensor/actuator signal processing?

Information based criteria are possible in the form of maximum entropy methods, but they often neglect other important metrics such as speed and robustness. Inversion models are also useful for signal processing.

5. Optimisation - What does it mean to be better? Optimisation methods identified include placement, maximum entropy, systems engineering, and selection.

6. Which specific sensors/actuators are suitable? Having reviewed the literature it is clear that no consensus can be found on the correct selection criteria to find the suitable sensors/actuators.

7. How do you define a good sensor/actuator? Different control metrics for control architecture analysis include: Degrees of freedom, closed loop performance metrics, open loop performance metrics and fault tolerance

8. Specific examples of where sensors/actuators are being used in industry?

Numerous examples have been reviewed including wireless sensors/actuators in communications, exoskeletons for disabled people, virtual actuators for power systems, and traditional automotive after-treatment sensors.

Dealing with nonlinearities within the engine system is an important area of work that determines the appropriate selection criteria to be used. System modelling can help with this, but the impact on the performance of the configuration requires optimisation methods. The literature suggests that optimisation can be split hierarchically. On the lower level is the selection and location of the sensors. At a higher level the optimisation is based on systems engineering, object oriented architecture and multi-criteria algorithms, such as the MOGA developed by Sheffield University. One of the critical questions to be considered early in the design phase is the approach to the optimisation; will it be a top-down approach or bottom-up? This requires in depth technical analysis of the system to determine. Architecture optimisation is an issue within engineering in general, particularly due to hierarchal considerations. The cost functions are dependent on low level optimisation and any useful assessment metrics are dependent on time dependent root mean square deviations. Thus, we have not only considered optimization based on technical metrics (i.e. robustness) but also on non-technical metrics (i.e. reliability). There are many metrics currently being used to assess sensor and actuator configurations as physical measures, linear algebra metrics and modern control metrics were all being used by different groups. There are several metrics that can be used to assess the quality of the system; however, the choice is dependent on the type of controller and sensors/actuators used. As many of the metrics were applied to network and signal based systems, we could use this to determine a robust information based control architecture by considering the system structure and how signals flow within the engine. This could be achieved by using maximum entropy methods from optimisation. Alternatively, the engine could be modelled as a sensor network made up of the signals that are contained within the control architecture. Sensors could then be selected to make a robust information centric configuration, which could make full use of the information available within the architecture. Using these methods, virtual instrumentation, as proposed in the literature [15] [51], could be applied in the information centric configuration.

Currently there is no clear consensus of selection criteria for specific sensors/actuators for automotive applications to determine an optimal configuration. Still, the literature does present many potential methods for determining the appropriate selection methods. Despite many methods used are based on determining the optimal placement, Page 13 of 16 it is suggested that maximum-entropy methods can give us a deeper understanding of our system from an information based perspective. The issue with entropy as a metric is it does not suggest the usefulness of the information. The assessment metrics are limited in value and use, but by using a combination of them, different levels of trade-offs can be achieved. However, for a thorough treatment, nonlinear dynamics and uncertainties need to be considered together, which requires sophisticated (non-Gaussian) stochastic models to establish the value of a control architecture. If achieved, optimal selection criteria of sensor and actuator configurations could reduce manufacturing costs by potentially reducing the number of sensors/actuators as demonstrated in the literature [20]. Nevertheless, for an accurate idea of cost savings, in-depth technical assessment would need to be carried out as it is difficult to measure without context and experimental validation. A method or a tool that can suggest through relevant assessment metrics the value of a control architecture to determine an optimal sensor and actuator configuration would be particularly useful for control design in many industries.

\section{Bibliography}

1. Onen, U., Botsali, F., Kalyoncu M., Tinkir, M. et al "Design and Actuator Selection of a Lower Extremity Exoskeleton," IEEE/ASME Transactions on Mechatronics, no. 2 (2014): 62332., 2014, doi:10.1109/TMECH.2013.2250295

2. Blevins, T., Chen, D., Han, S., Nixon, M. et al, "Process Control over Real-Time Wireless Sensor and Actuator Networks," Proceedings of the 2015 IEEE 17th International Conference on High Performance Computing and Communications, USA, Aug 24-26, 2015, doi:10.1109/HPCC-CSS-ICESS.2015.141

3. Puliyatoke, S., and Balasubramaniam, K., "A Novel Use of Signal Processing Tools for Fault Detection in IC Engines," Proceedings of the 2013 IEEE International Ultrasonics Symposium (IUS), Czech Republic, Jul 21-25, 2013, doi:10.1109/ULTSYM.2013.0116

4. Xiaojing, J., Xiaoqiang, Y., and Xiaolong W., "Networked Testing Platform of Internal Combustion Engine Based on Virtual Instrumentation," Proceedings of the 2012 Second International Conference on Intelligent System Design and Engineering Application, China, Jan 6-7, 2012, doi:10.1109/ISdea.2012.603

5. Mohanty, S., Gupta, K., Raju K., Mishra, V. et al "Characterization of Wireless Accelerometer Sensor and its Industrial Applications," Proceedings of the $201420^{\text {th }}$ National Conference on Communications (NCC), India, 28 Feb-2 Mar, 2014. doi:10.1109/ncc.2014.6811373.

6. Raoufat, M., Tomsovic, K., and Djouadi S., "Virtual Actuators for Wide-Area Damping Control of Power Systems," IEEE Transactions on Power Systems 31, no. 6 (2016): 4703-711. doi:10.1109/tpwrs.2015.2506345.

7. Husted, H., Roth, G., Nelson, S., Hocken, L. et al., "Sensing of Particulate Matter for On-Board Diagnosis of Particulate Filters," SAE Int. J. Engines 5(2):235-247, 2012, https://doi.org/10.4271/2012-01-0372.

8. Alfaya, J., Bejarano, G., Ortega, M., and Rubio, F., "Controllability Analysis and Robust Control of a One-Stage Refrigeration System," European Journal of Control 26 (2015): 53-62. doi:10.1016/j.ejcon.2015.08.001.

9. Franze, G., Tedesco, F., and Famularo, D., “Actuator Fault Tolerant Control: A Receding Horizon Set-Theoretic Approach," IEEE Transactions on Automatic Control 60, no. 8 (2015): 2225-230. doi:10.1109/tac.2014.2375731. 
10. Munz, U., Pfister, M., and Wolfrum, P., "Sensor and Actuator Placement for Linear Systems Based on H-2 and H- $\infty$ Optimization," IEEE Transactions on Automatic Control 59, no. 11 (2014): 2984-989. doi:10.1109/tac.2014.2351673.

11. Lee, H., Park, Y., and Park, Y-s., "Quantitative Measures of Compensation Capabilities and Output Noise Sensitivities of Linear Systems," Proceedings of the 2011 11th International Conference on Control, Automation and Systems, South Korea, 26-29 Oct, 2011

12. Lee, H., and Park, Y., "Quantitative Measures of Output Noise Sensitivities of Linear Systems in Modal Domain," Proceedings of the 2012 12th International Conference on Control, Automation and Systems, South Korea, 17-21 Oct, 2012

13. Ahmed, Q., Iqbal Bhatti, A., and Iqbal, M., "Virtual Sensors for Automotive Engine Sensors Fault Diagnosis in Second-Order Sliding Modes," IEEE Sensors Journal 11, no. 9 (2011): 1832840. doi:10.1109/jsen.2011.2105471.

14. Meza-Aguilar, M., Sanchez-Torres, J. D., Loukianov, A., Navarrete-Guzman, A. et al, "Observer Based Controller for Internal Combustion Engine," Proceedings of the 2013 10th International Conference on Electrical Engineering, Computing Science and Automatic Control (CCE), Mexico, 30 Sep-4 Oct, 2013. doi: $10.1109 /$ iceee.2013.6676067.

15. Jingping, L., Xiaolang, X., Yong, W., and Hanqian, Y., "Development of a Fast Response High Accuracy Virtual Air Flow Meter for Internal Combustion Engine Applications," Proceedings of the 2011 Third International Conference on Measuring Technology and Mechatronics Automation, China, 6-7 Jan, 2011. doi:10.1109/icmtma.2011.260.

16. Vaidya, U., Rajaram, R., and Dasgupta, S., "Actuator and Sensor Placement in Linear Advection PDE," Proceedings of the 2011 IEEE Conference on Decision and Control and European Control Conference, USA, 12-15 Dec, 2011. doi:10.1109/cdc.2011.6161350.

17. Lee, H., and Park, Y., "Quantitative Measures of Controllability for Descriptor systems," Proceedings of the 2013 13th International Conference on Control, Automation and Systems, South Korea, 20-23 Oct, 2013, doi:10.1109/ICCAS.2013.6704061.

18. Ma, J., Zhu, G. G., and Schock, H., "Adaptive Control of a Pneumatic Valve Actuator for an Internal Combustion Engine," IEEE Transactions on Control Systems Technology 19, no. 4 (2011): 730-43. doi:10.1109/tcst.2010.2054091.

19. Astrom, K.j., and B. Wittenmark. "A Survey of Adaptive Control Applications," Proceedings of the 1995 34th IEEE Conference on Decision and Control. doi:10.1109/cdc.1995.478986.

20. Cheng, Q., Jing-Hui, D., Jian-Ping, H., Ai-Min, L. et al, "Optimization Selection Approach for Distribution of Actuators in Active Vibration Control of Helicopter," Proceedings of the 2015 34th Chinese Control Conference (CCC), China, 28-30 Jul, 2015. doi:10.1109/chicc.2015.7260140.

21. Armaou, A., and Demetriou, M. A., "Towards Optimal Actuator Placement for Dissipative PDE Systems in the Presence of Uncertainty," Proceedings of the 2010 American Control Conference, USA, 30 Jun-2Jul, 2010. doi:10.1109/acc.2010.5531128.

22. Yoo, J., and Kim, H. J., "Optimal Sensor Placement for RSSbased Localization using Gaussian Process," Proceedings of the 2014 4th IEEE International Conference on Cyber Technology in Automation, Control and Intelligent, China, 4-7 Jun, 2014. doi:10.1109/cyber.2014.6917461.

Page 14 of 16
23. Skelton, R. E., and Li, F., "Economic Sensor/Actuator Selection and its Application to Flexible Structure Control," Proceedings of the Smart Structures and Materials 2004: Modeling, Signal Processing, and Control, 2004. doi:10.1117/12.540156.

24. Zhao, X., and Rinderknecht, S., "Investigation of Actuator Placement Approaches for Active Vibration Control in the Aircraft Engine," Proceedings of the 2015 IEEE Aerospace Conference, USA, 7-14 Mar, 2015. doi:10.1109/aero.2015.7119298.

25. Osegueda, R., Ferregut, C., George, M. J., Gutierrez, J. M. et al, "Maximum Entropy Approach to Optimal Sensor Placement for Aerospace Non-Destructive Testing," Maximum Entropy and Bayesian Methods, 1998, 277-89. doi:10.1007/978-94-0115028-6_23.

26. Kudikala, R., Mills, A. R., Fleming, P. J., Tanner, G. F. et al, "Real World System Architecture Design Using Multi-Criteria Optimization: A Case Study," EVOLVE - A Bridge between Probability, Set Oriented Numerics, and Evolutionary Computation IV Advances in Intelligent Systems and Computing, 2013, 245-60. doi:10.1007/978-3-319-01128-8 16.

27. Moreno, C. P., Pfifer, H., and Balas, G. J, "Actuator and Sensor Selection for Robust Control of Aeroservoelastic Systems," Proceedings of the 2015 American Control Conference (ACC), USA, 1-3 Jul, 2015. doi:10.1109/acc.2015.7171010.

28. Huber, J., Kopecek, H., and Hofbaur, M., "Sensor Selection for Fault Parameter Identification Applied to an Internal Combustion Engine," Proceedings of the 2014 IEEE Conference on Control Applications (CCA), France, 8-10 Oct, 2014. doi:10.1109/cca.2014.6981334.

29. Bertrand, A., and Moonen, M., "Distributed Adaptive Eigenvector Estimation of the Sensor Signal Covariance Matrix in a Fully Connected Sensor Network," Proceedings of the 2013 IEEE International Conference on Acoustics, Speech and Signal Processing, Canada, 26-31 May, 2013. doi:10.1109/icassp.2013.6638458.

30. Sequeira, J., Tsourdos, A., and Lazarus, S. B., "Robust Covariance Estimation for Data Fusion from Multiple Sensors," IEEE Transactions on Instrumentation and Measurement 60, no. 12 (2011): 3833-844. doi:10.1109/tim.2011.2141230.

31. Li, X., Yu, H., Zeng, P., and Sun, L., "Study on Robust Stability Inverse Problem for Linear Systems," Proceedings of the 2014 CACS International Automatic Control Conference (CACS 2014), Taiwan, 26-28 Nov, 2014. doi:10.1109/cacs.2014.7097158.

32. Franchi, A., Petitti, A., and Rizzo, A., "Distributed Estimation of the Inertial Parameters of an Unknown Load via Multi-Robot Manipulation," Proceedings of the 53rd IEEE Conference on Decision and Control, USA, 15-17 Dec, 2014. doi:10.1109/cdc.2014.7040346.

33. Cochran, D., Howard, S. D., Moran, B., and Schmitt, H. A., "Maximum-Entropy Surrogation in Network Signal Detection," Proceedings of the 2012 IEEE Statistical Signal Processing Workshop (SSP), USA, 5-8 Aug, 2012. doi:10.1109/ssp.2012.6319686.

34. Rajasegarar, S., Leckie, C., and Palaniswami, M., "SpatioTemporal Estimation with Bayesian Maximum Entropy and Compressive Sensing in Communication Constrained Networks," Proceedings of the 2014 IEEE International Conference on Communications (ICC), Australia, 10-14 Jun, 2014. doi:10.1109/icc.2014.6884036.

35. Dai, J., and Lin, H., "Learning-based Design of Fault-Tolerant Cooperative Multi-Agent Systems," Proceedings of the 2015 
American Control Conference (ACC), USA, 1-3 Jul, 2015. doi:10.1109/acc.2015.7171015.

36. Machado, J.a., "Exploiting Sensor Redundancy for the Calculation of Fractional Derivatives in the Presence of Noise," Signal Processing 92, no. 1 (2012): 204-09. doi:10.1016/j.sigpro.2011.07.007.

37. Hammerschmidt, D. and Leteinturier, P., "Automotive Sensors \& Sensor Interfaces," SAE Technical Paper 2004-01-0210, 2004, https://doi.org/10.4271/2004-01-0210.

38. McKelvey, T., Andersson, I., and Thor, M., "Estimation of Combustion Information by Crankshaft Torque Sensing in an Internal Combustion Engine," Proceedings of the 2007 2nd IEEE International Workshop on Computational Advances in Multi-Sensor Adaptive Processing, USA, 12-14 Dec, 2007. doi:10.1109/camsap.2007.4497979.

39. Balau, A. E., Caruntu, C. F., Patrascu, D. I., Lazar, C. et al, "Modeling of a Pressure Reducing Valve Actuator for Automotive Applications," Proceedings of the 2009 IEEE International Conference on Control Applications, Russia, 8-10 Jul, 2009. doi:10.1109/cca.2009.5280939.

40. Aono, T., and Kowatari, T., "A Signal Processing Algorithm for Compensating for Back Flow Effect in Intake Air Mass Measurement in Internal Combustion Engines," Proceedings of the 2004 IEEE International Conference on Control Applications, 2-4 Sep, 2004. doi:10.1109/cca.2004.1387237.

41. Philips, P., Van de Wal, M., de Jager, B., "Selection of Sensors and Actuators based on a Necessary Condition for Robust Performance," Proceedings of the 1997 European Control Conference, Belgium, 1-7 Jul, 1997

42. Rotea, M.a., Corless, M., Da, D., and Petersen, I.r., "Systems with Structured Uncertainty: Relations between Quadratic and Robust Stability," IEEE Transactions on Automatic Control 38, no. 5 (1993): 799-803. doi:10.1109/9.277250.

43. Pandolfi, L., and Olbrot, A.W., "The Effects of Multiple Right Half Plane Zeros and Time Delays on the Achievable Sensitivity Reduction," Proceedings of the 29th IEEE Conference on Decision and Control, USA, 5-7 Dec, 1990. doi:10.1109/cdc.1990.203897.

44. Cheng, V., and Desoer, C., "Limitations on the Closed-Loop Transfer Function due to Right-Half Plane Transmission Zeros of the Plant," IEEE Transactions on Automatic Control 25, no. 6 (1980): 1218-220. doi:10.1109/tac.1980.1102530.

45. Norris, G., Skelton, R., "Sensor and Actuator Selection for Optimal Closed-Loop Performance in the Presence of Correlated Noise," Proceedings of the American Control Conference, USA, 10-12 Jun, 1987

46. Li, A., and Song, L., "Multisensor Correlation Analysis and its Application in Coal Mines," Proceedings of the 2009 WRI Global Congress on Intelligent Systems, China, 19-21 May, 2009. doi:10.1109/gcis.2009.79.

47. Welsh, J., and Rojas, C., "Frequency Localising Basis Functions for Wide-band System Identification: A Condition Number Bound for Output Error Systems," Proceedings of the 2007 European Control Conference, Greece, 2-5 Jul, 2007

48. Seron, M. M., and De Dona, J. A., "Fault Tolerant Control using Virtual Actuators and Invariant-Set based Fault Detection and Identification," Proceedings of the 48th IEEE Conference on Decision and Control (CDC) held jointly with 2009 28th Chinese Control Conference, China, 15-18 Dec, 2009. doi:10.1109/cdc.2009.5399909.

49. Hoblos, G., Staroswiecki, M., and Aitouche, A., "Optimal Design of Fault Tolerant Sensor Networks," Proceedings of the 2000 IEEE International Conference on Control Applications,
USA, 27 Sep, 2000, Conference Proceedings (Cat. No.00CH37162). doi:10.1109/cca.2000.897468.

50. Chen, T.1., and You, R.z., "A Novel Fault-Tolerant Sensor System for Sensor Drift Compensation," Sensors and Actuators A: Physical 147, no. 2 (2008): 623-32. doi:10.1016/j.sna.2008.05.026.

51. Richter, J.h., Lunze, J., and Schlage, T., "Control Reconfiguration of a Thermofluid Process by Means of a Virtual Actuator," IET Control Theory \& Applications 1, no. 6 (2007): 1606-620. doi:10.1049/iet-cta:20060506.

52. Chevrel, P., and Guo, L., "Actuator and Sensor Placement using Convex Optimization Tools," Proceedings of the 2001 European Control Conference, Portugal, 4-7 Sep, 2001

53. Antoniades, C., and Christofides, P.d., "Integrated Optimal Actuator Placement and Robust Control of Uncertain TransportReaction Processes," Proceedings of the 2001 American Control Conference. USA, 25-27 Jun, (Cat. No.01CH37148), 2001. doi:10.1109/acc.2001.946025.

54. Canxing, L., Xingshan, L., Ping, Z., and Jing, D., "A Review on Optimal Sensor Placement for Health Monitoring," Proceedings of the 2007 8th International Conference on Electronic Measurement and Instruments, China, 16-18 Aug, 2007, doi:10.1109/icemi.2007.4351109.

55. Demetriou, M.a., and Armaou, A., "Optimal Actuator Placement and Model Reduction for a Class of Parabolic Partial Differential Equations using Spatial $\mathrm{H}_{2}$ norm," Proceedings of the 2005, American Control Conference, USA, 8-10 Jun, 2005, doi:10.1109/acc.2005.1470716.

56. Chmielewski, D.j., and Peng, J.K., "Covariance-based Hardware Selection-Part I: Globally Optimal Actuator Selection," IEEE Transactions on Control Systems Technology 14, no. 2 (2006): 355-61. doi:10.1109/tcst.2005.863670.

57. Peng, J.K., and Chmielewski, D.j., "Covariance-based Hardware Selection-Part II: Equivalence Results for the Sensor, Actuator, and Simultaneous Selection Problems," IEEE Transactions on Control Systems Technology 14, no. 2 (2006): 362-68. doi:10.1109/tcst.2005.863671.

58. Vanbeveren, Y., and Gevers, M., "On Optimal and Suboptimal Actuator Selection Strategies," IEEE Transactions on Automatic Control 21, no. 3 (1976): 382-85. doi:10.1109/tac.1976.1101237.

59. Lynch, P.M., De Paso, J., "An Object Oriented Intelligent Control Architecture," Proceedings of the 1992 American Control Conference, USA, 24-26 Jun, 1992

60. Voulgaris, P.G. "Optimal Control of Systems with Delayed Observation Sharing Patterns via Input-Output Methods," Proceedings of the 39th IEEE Conference on Decision and Control, Australia, 12-15 Dec, 2000 (Cat. No.00CH37187). doi:10.1109/cdc.2000.914143.

61. Clarhaut, J., Cocquempot, V., Conrard, B., and Hayat, S., "Optimal Design of Dependable Control System Architectures Using Temporal Sequences of Failures," IEEE Transactions on Reliability 58, no. 3 (2009): 511-22. doi:10.1109/tr.2009.2026790.

62. Freudenberg, J., and Looze, D., "Right Half Plane Poles and Zeros and Design Tradeoffs in Feedback Systems," IEEE Transactions on Automatic Control 30, no. 6 (1985): 555-65. doi:10.1109/tac.1985.1104004.

63. Bushnell, M.J., "Application of Object Oriented Techniques in a Modular Expert System Architecture for Transmission System Monitoring and Control," Proceedings of the 1994 International Conference on Control, UK, 21-24 Mar, 1994. doi:10.1049/cp:19940121.

Page 15 of 16 
64. Chan, F.T.S., Zhang, J., Lau, H.C.W., and Ning, A., "ObjectOriented Architecture of Control System for Agile Manufacturing Cells." Proceedings of the 2000 IEEE International Conference on Management of Innovation and Technology. ICMIT 2000. Management in the 21st Century, Singapore, 12-15 Nov, 2000, (Cat. No.00EX457). doi:10.1109/icmit.2000.916819.

65. Papadimitropoulos, A., Rovithakis, G. A., and Parisini, T., "Fault Detection in Mechanical Systems with Friction Phenomena: An Online Neural Approximation Approach," IEEE Transactions on Neural Networks 18, no. 4 (2007): 1067-082. doi:10.1109/tnn.2007.899182.

66. Sarrate, R., Puig, V., Escobet, T., and Rosich, A., "Optimal Sensor Placement for Model-based Fault Detection and Isolation," Proceedings of the 2007 46th IEEE Conference on Decision and Control, USA, 12-14 Dec, 2007. doi:10.1109/cdc.2007.4434452.

67. Zhang, Y.M., and Kovacevic, R., "Robust Control of Interval Plants: A Time Domain Method," IEE Proceedings - Control Theory and Applications 144, no. 4 (1997): 347-53. doi:10.1049/ip-cta:19971170.

68. Van De Wal, M., and De Jager, B., "A Review of Methods for Input/Output Selection,” Automatica 37, no. 4 (2001): 487-510. doi:10.1016/s0005-1098(00)00181-3.

69. Choi, M., "Exhaust processing device control method for vehicle," KR Patent KR20150122935 (A), November 03, 2015.

70. Seiberlich, M., McNulty, M., Rodriguez, J., and Schipper, J., "Setpoint Bank Control Architecture," U.S. Patent US2013197779 (A1), August 01, 2013.

71. Matohara, S., Kumano, K., and Akagi, Y., "Control Device of Internal Combustion Engine,” U.S. Patent US2016215749 (A1), July 28, 2016.

72. Fischer, W., and Kluth, C., "Method for Processing a Signal of a Combustion Chamber Pressure Sensor," KR Patent KR20160056822 (A), issued May 20, 2016.

73. Gustmann, M., Fehrmann, R., Heise, M., Saravanalingnam, A., and Selle, R., "Method and Apparatus for Controlling a Reciprocating-Piston Engine Having Several Cylinders," US Patent US2016237933 (A1), August 18, 2016.

74. Amano, M., Sakamoto, S., Hirayama, T., and Sasayama, T., "Signal Processing Method for Hot-Wire Flow Rate Sensor," JP Patent JPS5895214 (A), June 06, 1983.

75. Berns, A., Daetz, M., Hahn, H., and Pelz, N., "Controller and Method for Controlling a NOx-Sensor Arranged in an Exhaust Gas Channel of an Internal Combustion Engine," EP Patent EP1413728 (A2), April 28, 2004.

\section{Contact Information}

Mr Rhys Comissiong

Aeronautical and Automotive Engineering

Stewart Miller Building

Loughborough University

Leicestershire

LE11 3TU

r.m.comissiong@1boro.ac.uk

\section{Acknowledgements}

This work was funded as part of the EPSRC Centre for Doctoral Training in Embedded Intelligence grant number EP/L014998/1.

Page 16 of 16

$30 / 01 / 2018$ 